\title{
Review
}

\section{Progesterone receptors - animal models and cell signaling in breast cancer Role of steroid receptor coactivators and corepressors of progesterone receptors in breast cancer}

\author{
Xiuhua Gao and Zafar Nawaz
}

Department of Molecular and Cellular Biology, Baylor College of Medicine, Houston, Texas, USA

Correspondence: Zafar Nawaz, Department of Molecular and Cellular Biology, Baylor College of Medicine, One Baylor Plaza, Houston, TX 77030, USA. Tel: +1 713798 6258; fax: +1 713790 1275; e-mail: zn029815@bcm.tmc.edu

\begin{abstract}
Progesterone, an ovarian steroid hormone, plays a key role in the development and function of the mammary gland, as it also does in the uterus and the ovary. The action of progesterone is mediated through its intracellular cognate receptor, the progesterone receptor (PR), which functions as a transcription factor that regulates gene expression. As with other nuclear receptors, coregulators (coactivators and corepressors) recruited by the liganded or unliganded PR, either to enhance or to suppress transcription activity, modulate the function of the PR. Mutation or aberrant expression of the coregulators might thus affect the normal function of the PR and hence disrupt the normal development of the mammary gland, which may lead to breast cancer.
\end{abstract}

Keywords: breast cancer, coactivator, corepressor, progesterone receptor

\section{Introduction}

The progesterone receptor (PR) is a member of the nuclear receptor superfamily, which specifically regulates the expression of target genes in response to the hormonal stimulus. In the absence of progesterone, the PR is sequestered in a nonproductive form associated with heat shock proteins and other cellular chaperones. In this state, the PR is considered unable to influence the rate of transcription of its cognate promoters [1]. On binding with progesterone, the PR undergoes a series of events, including conformational changes, dissociation from heat shock protein complexes, dimerization, phosphorylation, and nuclear translocation, which enables its binding to progesterone-response elements within the regulatory regions of target genes. The binding of PR to the progesterone-response elements is followed by the recruitment of coactivators and the basal transcription machinery, leading to the upregulation of target gene transcription.

The PR exists as two isoforms in most rodents and humans, PR-A and PR-B, which are produced from a single gene by translation initiation at two distinct start codons under the control of separate promoters [2]. The difference between PR-A and PR-B is that PR-A is a truncated form of PR-B. In humans, the N-terminal 164 amino acids of PR-B are missing in isoform PR-A. Detailed molecular dissection has identified two distinct activation function domains ( $\mathrm{AFs}$ ) within both PRs: AF-1, which is located in the $\mathrm{N}$-terminal region, is ligand independent; AF-2, which is ligand dependent, is contained in the ligand-binding domain that is located in the $\mathrm{C}$-terminal region. A DNA-binding domain and the hinge region are

$\mathrm{AF}=$ activation function domain; $\mathrm{CBP}=\mathrm{CREB}$-binding protein; $\mathrm{E} 6-\mathrm{AP}=\mathrm{E} 6$-associated protein; $\mathrm{ER}=$ estrogen receptor; $\mathrm{N}$-CoR $=$ nuclear receptor corepressor; $\mathrm{PR}=$ progesterone receptor; RPF1 = receptor potentiation factor-1; RTA = repressor of tamoxifen transcriptional activity; SMRT = silencing mediator of retinoid and thyroid receptor; SRA = steroid receptor RNA activator; SRC = steroid receptor coactivator; Uba3 = ubiquitinactivating enzyme. 
Figure 1

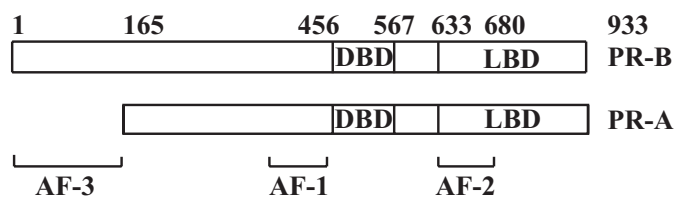

Schematic representation of the progesterone receptor PR-A and PR$B$ proteins. The DNA-binding domain (DBD), the ligand-binding domain (LBD) and activation function domains (AFs) are indicated.

mapped to the central region of both receptors. Furthermore, a unique activation function domain, AF-3, is contained in the upstream segment of PR-B that is missing in PR-A (Fig. 1).

Progesterone and estrogen are essential regulators of female reproductive activity. Through their cognate receptors, estrogen and progesterone regulate the normal development of the ovary, the uterus and the mammary gland, and play key roles in the tumorigenesis of these tissues. It has been demonstrated by estrogen receptor (ER) and PR knockout mice that estrogen controls the early ductal morphogenesis of the mammary gland, whereas progesterone controls ductal branching and alveolar development of the mammary gland during pregnancy [3].

Although the two forms of the PR have similar structures and are identical in DNA and ligand binding, in vitro studies using a reconstituted progesterone-responsive transcription system in mammalian cells revealed that PR-A and PR-B are not functionally identical. In most cases, PR-B acts as a potent activator of transcription of target genes, whereas PR-A acts as a dominant repressor of transcription of PR-B as well as a few other nuclear receptors [4]. The AF-3 domain in PR-B is partially responsible for the higher transcriptional activity of PR-B relative to PR-A. Moreover, an inhibitory function domain that is located in the $\mathrm{N}$-terminus of both receptor isoforms has been identified [2]. This inhibition function domain can inhibit the activity of AF-1 and AF-2 but not that of AF-3, which explains why PR-B is a potent activator of transcription. The inhibition function domain is functionally independent and is transferable; when placed upstream of the ER, the inhibition function domain can also suppress ER activity.

The relative expression of PR-A and PR-B in the target tissues is dependent on species, cellular context, and the physiological and hormonal status. The ratio of PR-A to PR-B in specific tissues or cell types defines the physiological and pharmacological responses to progesterone. In the mammary gland, the ratios of PR-A to PR-B are constant from puberty to pregnancy, although there are species differences [5]. From PR-A knockout mice, it has been demonstrated that PR-B is mainly responsible for the normal proliferative and differentiative responses of the mammary gland to progesterone, because PR-A knockout mice exhibit a similar phenotype to PR knockout mice [3]. Overexpression of PR-A over PR-B in transgenic mice results in extensive epithelial cell hyperplasia, in excessive ductal branching, and in a disorganized basement membrane. All these features are associated with neoplasia [6]. Furthermore, the development of the mammary gland in PR-B overexpressed transgenic mice is also abnormal [7], indicating that a regulated expression of PR-A and PR-B and the native ratios of the two isoforms are critical to the appropriate responsiveness of the mammary gland to progesterone. Consistent with the findings from animal studies, very low levels of PR-B and a consequently high PR-A:PR-B ratio were found in a significant proportion of human breast cancer samples [8]. Taking these findings together, it can be inferred that imbalance of PR-A versus PR-B may be associated with the development, progression or prognosis of breast cancer.

Coactivators are factors that can interact with nuclear receptors in a ligand-dependent manner and enhance their transcriptional activity. Corepressors are factors that interact with nuclear receptors and repress their transcriptional activity. Both types of coregulators are required for efficient modulation of target gene transcription by the PR [9]. Changes in the expression level and pattern of PR coactivators or corepressors, or mutation of their function domains, might therefore affect the transcriptional activity of the PR and hence cause disorders of its target tissues, including the mammary gland. The present review will describe the coactivators and corepressors that are involved in the transcriptional modulation of PRs, with emphasis on their roles in breast cancer development and progression.

\section{Progesterone receptor coactivators The steroid receptor coactivator family}

The steroid receptor coactivator (SRC) family is composed of three distinct but structurally and functionally related members: SRC-1 (nuclear receptor coactivator 1), SRC-2 (transcription intermediary factor $2 /$ glucocorticoid receptor-interacting protein 1 /nuclear receptor coactivator 2 ), and SRC-3 (p300/CREB-binding protein [CBP] cointegrator-associated protein/receptor-associated coactivator 3/activator of thyroid and retinoid receptors/amplified in breast cancer 1 /thyroid receptor activator molecule 1 ). SRC-1 was the first identified coactivator for the steroid receptor superfamily, which was cloned and characterized in 1995 [10]. SRC-2 and SRC-3 were then identified thereafter by several laboratories [9].

Sequence analysis of SRC proteins identified a basic helix-loop-helix domain and two Per-Arnt-Sim domains in the amino-terminal region. The basic helix-loop-helix/ 
Per-Arnt-Sim domain is highly conserved among the SRC members, and it serves as a DNA binding and protein dimerization motif in many transcription factors [11]. Following the basic helix-loop-helix/Per-Arnt-Sim domain, there are a centrally located receptor-interacting domain and a C-terminal transcriptional activation domain. Detailed analysis revealed three conserved LXXLL motifs (nuclear receptor box) in the receptor-interacting domain, which appear to contribute to the specificity of coactivatorreceptor interaction. Histone acetyltransferase activity was identified in the C-terminal region of SRC members, and there also exist activation domains that can interact with the CBP.

All three members of the SRC family interact with the PR and enhance its transcriptional activation in a ligand-dependent manner $[12,13]$. Targeted deletion of the SRC-1 gene in mice has indicated that SRC-1 is important for the biological actions of progesterone in mammary gland development since the hormone-induced ductal elongation and alveolar development is greatly impaired in the null mice [14]. In the meantime, the expression of SRC-2 mRNA was elevated in SRC-1 null mice, suggesting that SRC-2 can partially compensate for SRC-1 function [14].

SRC-3 is the most distinct among the three members. It coactivates not only the nuclear receptors, but also other unrelated transcription factors such as those in the cAMP or cytokine pathways [15]. Compared with the widespread expression of SRC-1 and SRC-2, expression of SRC-3 is restricted to the mammary gland and several other tissues [16]. Disruption of the SRC-3 gene in mice causes severe growth and reproductive defects, including the retardation of mammary gland development [17]. Furthermore, amplification and overexpression of SRC-3 were observed in $10 \%$ and $64 \%$ of human primary breast cancers, respectively [18]. This observation indicates that SRC-3 is not only essential for the normal mammary development, but also plays a role in breast tumorigenesis.

\section{E6-associated protein/RPF1}

E6-associated protein (E6-AP) and RPF1, the human homolog of yeast RSP5, are E3 ubiquitin-protein ligases that target proteins for degradation by the ubiquitin pathway. They are also characterized as coactivators of steroid receptors. It has been demonstrated by transient transfection assay that RPF1 and E6-AP could potentiate the ligand-dependent transcriptional activity of the PR, the glucocorticoid receptor, and other nuclear receptors $[19,20]$. Furthermore, RPF1 and E6-AP acted synergistically to enhance PR transactivation [20]. Additionally, the coactivation functions of E6-AP and RPF1 are separable from the E3 ubiquitin-protein ligase activity, as ubiquitin ligase-defective E6-AP and RPF1 exhibited normal coacti-
E6-AP is expressed in many tissues, including the mammary gland. From its ability to coactivate the PR and the ER in a hormone-dependent manner, it was assumed that E6-AP is an essential regulator for the development of normal mammary gland and mammary tumors. The first evidence of a relationship between E6-AP and breast cancers was obtained from the study of a spontaneous mouse mammary tumorigenesis model, which demonstrated that E6-AP was overexpressed in tumors when compared with normal tissues [21].

We recently examined the expression pattern of E6-AP in biopsy samples of human breast cancers, and our results showed that E6-AP expression was decreased in tumors in comparison with the adjacent normal tissues (Gao et al., unpublished data, 2002). Furthermore, we demonstrated that the decreased expression of E6-AP was stage dependent, and that the expression of E6-AP was inversely correlated with that of the ER in breast tumors. Since the ER plays a major role in breast cancer development and the $\mathrm{PR}$ is a target of estrogen, the changes of the expression level of E6-AP might interfere with the normal functioning of the ER and the PR. Hence, E6-AP may participate in the formation and progression of breast tumors.

\section{Steroid receptor RNA activator}

The growing family of nuclear receptor coactivators has recently acquired a unique member, steroid receptor RNA activator (SRA) [22]. Differing from the other coactivators, SRA functions as a RNA transcript instead of as a protein. SRA specifically coactivates the transcriptional activity of steroid receptors, including the PR, the ER, the glucocorticoid receptor, and the androgen receptor. It has been demonstrated that SRA exists in a ribonucleoprotein complex containing SRC-1 and that it mediates transactivation through the AF-1 domain located at the $\mathrm{N}$-terminal region of nuclear receptors, distinguishing it from the other coactivators.

SRA is expressed in normal and malignant human mammary tissues $[23,24]$. An elevated expression of SRA was found in tumors compared with the adjacent normal region [24]. Although it is currently unknown whether the expression of SRA is correlated with that of the PR or the $E R$, the increase in the SRA levels in tumor cells may contribute to the altered ER/PR action that is known to occur during breast tumorigenesis.

\section{L7/SPA}

L7/SPA is a $27 \mathrm{kDa}$ protein containing a basic leucine zipper domain. L7/SPA is an antagonist-specific transcriptional coactivator because it can only potentiate the partial agonist activity of some antagonists, including tamoxifen and RU486, but has no effect on the agonist-mediated transcription [25]. This unique property of L7/SPA suggests that it might play a role in the development of resistance to hormone therapy for breast cancers. 


\section{CBP/p300}

CBP was initially characterized as a coactivator required for efficient transactivation of cAMP-response elementbinding protein, and p300 was first identified as a coactivator of the adenovirus E1A oncoprotein. CBP and p300 share many functional properties: both of them function as coactivators for multiple nuclear receptors as well as p53 and nuclear factor- $\mathrm{KB}$ [9], both possess intrinsic histone acetyltransferase activity, and both can recruit histone acetyltransferase and CBP/p300-associated factor [26]. Besides, CBP/p300 interacts with members of the SRC family and synergizes with SRC-1 in the transactivation of the ER and the PR [27].

\section{Other coactivators}

In addition to the coactivators already discussed, there are a few other proteins that have been demonstrated to upregulate the transcriptional activity of the PR. Chromatin highmobility group protein 1, chromatin high-mobility group protein 2, TIP60 (Tat-interacting protein), proline-rich nuclear receptor coregulatory protein 1, proline-rich nuclear receptor coregulatory protein 2, Cdc25B, and GT198 all function as PR coactivators, as demonstrated by transient transfection assays [28-32]. Cdc25B is prominent among these coactivators in terms of its roles in breast cancer development, because Cdc25B transgenic mice exhibit mammary gland hyperplasia and increased steroid hormone responsiveness [31]. The significance of all these coactivators in vivo needs to be further investigated.

\section{Progesterone receptor corepressors Nuclear receptor corepressor/silencing mediator of retinoid and thyroid receptors}

Nuclear receptor corepressor (N-CoR) and silencing mediator of retinoid and thyroid receptor (SMRT) are both corepressors of numerous transcription factors, including steroid hormone receptors. Both $\mathrm{N}-\mathrm{CoR}$ and SMRT interact with the nuclear receptors through the receptorinteracting domains located in the C-terminal portion of the proteins, while their transcriptional repression domains are mapped to the N-termini [33]. N-CoR and SMRT also associate with HDAC3 in large protein complexes, which is an important pathway for transcriptional repression. Corepressors N-CoR and SMRT interact with the nuclear receptors either in the absence of agonists (in the case of the thyroid receptor and the retinoid acid receptor) or in the presence of antagonists (in the case of steroid receptors) [33]. Since N-CoR and SMRT are common corepressors for transcription factors, slight alteration of their expression level in certain tissues might result in significant transcriptional changes, leading to altered development of the mammary gland, even tumors.

\section{BRCA1}

$B R C A 1$ is a breast cancer susceptibility gene, and its inherited mutations are correlated with an increased risk of breast cancer and ovarian cancer [34]. The role of $B R C A 1$ in cancer development is unclear. In addition to its ability to coactivate p53 and to modulate p300/CBP expression, BRCA1 is also a ligand-independent corepressor for the ER, the androgen receptor and the PR [35]. If $B R C A 1$ is mutated, all of these pathways will be more or less impaired. The effect of BRCA1 in cancer development might therefore be multiplex.

\section{Other corepressors}

Ubiquitin-activating enzyme 3 (Uba3) is the catalytic subunit of the activating enzyme in the ubiquitin-like NEDD8 (neural precursor cell-expressed developmentally downregulated) conjugation (neddylation) pathway. Uba3 was recently demonstrated as a corepressor of the ER, the androgen receptor and the PR in mammalian transfection assays [36]. Uba3 inhibited the transactivation of the ER in a time-dependent manner, and neddylation activity of Uba3 is required for this suppression. This suggests that Uba3 suppresses steroid receptor activity by promoting the termination of receptor-mediated gene transcription rather than by interfering with the initial events.

Repressor of tamoxifen transcriptional activity (RTA) has recently been defined as a potent repressor of tamoxifenmediated $\mathrm{ER} \alpha$ transcriptional activity as well as an agonist of the ER $\beta$, the glucocorticoid receptor, and the PR [37]. The interaction of RTA with the nuclear receptors requires the participation of RNA, because mutation of the RNA recognition motif in RTA compromises its ability to repress transcription [37].

The roles of Uba3 and RTA in mammary gland development and tumorigenesis await further study.

\section{Summary}

As a transcription factor, the PR activates target gene transcription in response to the hormonal stimulus, and its functions are modulated by coactivators and corepressors. Different coregulators exert their actions through different mechanisms, and involvement in the development of normal mammary gland and the formation or progression of tumors has been reported in some coactivators and some corepressors. The coactivators and corepressors of the PR so far identified are not PR specific, since they can also modulate the transactivation of many other nuclear receptors. In addition, no unique coregulators of PR-A or PR-B have been identified. Identification of PR-specific coregulatory proteins, especially PR-A interacting factors or PR-B interacting factors, is an important goal of future study.

\section{Acknowledgements}

The authors thank Dr Neil J McKenna for critical reading of this manuscript. This work was supported in part by grants to Dr Zafar Nawaz (NIH DK56833, DOD DAMD17-99-1-9075, and DOD 17-00-1-0142). 


\section{References}

1. McDonnell DP: Unraveling the human progesterone receptor signal transduction pathway. Trends Endocrinol Metab 1995, 6: 133-138.

2. Richer JK, Jacobsen BM, Manning NG, Abel MG, Wolf DM, Horwitz KB: Differential gene regulation by the two progesterone receptor isoforms in human breast cancer cells. $J$ Biol Chem 2002, 277:5209-5218.

3. Conneely OM, Lydon JP: Progesterone receptors in reproduction: functional impact of the A and B isoforms. Steroids 2000, 65:571-577.

4. Giangrande PH, Pollio G, McDonnell DP: Mapping and characterization of the functional domains responsible for the differential activity of the $A$ and $B$ isoforms of the human progesterone receptor. J Biol Chem 1997, 272:32889-32900.

5. Conneely OM, Lydon JP, De Mayo F, O'Malley BW: Reproductive functions of the progesterone receptor. J Soc Gynecol Investig 2000, 7(1 suppl):S25-S32.

6. Shyamala G, Yang X, Silberstein G, Barcellos-Hoff MH, Dale E: Transgenic mice carrying an imbalance in the native ratio of $A$ to $B$ forms of progesterone receptor exhibit developmental abnormalities in mammary glands. Proc Natl Acad Sci USA 1998, 95:696-701.

7. Shyamala G, Yang X, Cardiff RD, Dale E: Impact of progesterone receptor on cell-fate decisions during mammary gland development. Proc Natl Acad Sci USA 2000, 97:3044-3049.

8. Graham JD, Yeates C, Balleine RL, Harvey SS, Milliken JS, Bilous AM, Clarke CL: Progesterone receptor A and B protein expression in human breast cancer. J Steroid Biochem Mol Biol 1996, 56:93-98.

9. McKenna NJ, Lanz RB, O'Malley BW: Nuclear receptor coregulators: cellular and molecular biology. Endocr Rev 1999, 20: 321-344.

10. Onate SA, Tsai SY, Tsai MJ, O'Malley BW: Sequence and characterization of a coactivator for the steroid hormone receptor superfamily. Science 1995, 270:1354-1357.

11. Leo C, Chen JD: The SRC family of nuclear receptor coactivators. Gene 2000, 245:1-11.

12. Voegel JJ, Heine MJ, Zechel C, Chambon P, Gronemeyer H: TIF2, a $160 \mathrm{kDa}$ transcriptional mediator for the ligand-dependent activation function AF-2 of nuclear receptors. EMBO J 1996, 15:3667-3675

13. Li H, Gomes PJ, Chen JD: RAC3, a steroid/nuclear receptorassociated coactivator that is related to SRC-1 and TIF2. Proc Natl Acad Sci USA 1997, 94:8479-8484.

14. Xu J, Qiu Y, DeMayo FJ, Tsai SY, Tsai MJ, O'Malley BW: Partial hormone resistance in mice with disruption of the steroid receptor coactivator-1 (SRC-1) gene. Science 1998, 279:1922-1925

15. Torchia J, Rose DW, Inostroza J, Kamei Y, Westin S, Glass CK, Rosenfeld MG: The transcriptional co-activator p/CIP binds CBP and mediates nuclear-receptor function. Nature 1997, 387:677-684.

16. Suen CS, Berrodin TJ, Mastroeni R, Cheskis BJ, Lyttle CR, Frail DE: A transcriptional coactivator, steroid receptor coactivator3 , selectively augments steroid receptor transcriptional activity. J Biol Chem 1998, 273:27645-27653.

17. Xu J, Liao L, Ning G, Yoshida-Komiya H, Deng C, O'Malley BW: The steroid receptor coactivator SRC-3 (p/CIP/RAC3/AIB1/ ACTR/TRAM-1) is required for normal growth, puberty, female reproductive function, and mammary gland development. Proc Natl Acad Sci USA 2000, 97:6379-6384.

18. Anzick SL, Kononen J, Walker RL, Azorsa DO, Tanner MM, Guan XY, Sauter G, Kallioniemi OP, Trent JM, Meltzer PS: AIB1, a steroid receptor coactivator amplified in breast and ovarian cancer. Science 1997, 277:965-968.

19. Imhof MO, McDonnell DP: Yeast RSP5 and its human homolog hRPF1 potentiate hormone-dependent activation of transcription by human progesterone and glucocorticoid receptors. Mol Cell Biol 1996, 16:2594-2605.

20. Nawaz Z, Lonard DM, Smith CL, Lev-Lehman E, Tsai SY, Tsai MJ, O'Malley BW: The Angelman syndrome-associated protein, E6-AP, is a coactivator for the nuclear hormone receptor superfamily. Mol Cell Biol 1999, 19:1182-1189.

21. Sivaraman L, Nawaz Z, Medina D, Conneely OM, O'Malley BW: The dual function steroid receptor coactivator/ubiquitin protein-ligase integrator E6-AP is overexpressed in mouse mammary. Breast Cancer Res Treat 2000, 62:185-189.
22. Lanz RB, McKenna NJ, Onate SA, Albrecht U, Wong J, Tsai SY, Tsai MJ, O'Malley BW: A steroid receptor coactivator, SRA, functions as an RNA and is present in an SRC-1 complex. Cell 1999, 97:17-27.

23. Leygue E, Dotzlaw H, Watson PH, Murphy LC: Expression of the steroid receptor RNA activator in human breast tumors. Cancer Res 1999, 59:4190-4193.

24. Murphy LC, Simon SL, Parkes A, Leygue E, Dotzlaw H, Snell L, Troup S, Adeyinka A, Watson PH: Altered expression of estrogen receptor coregulators during human breast tumorigenesis. Cancer Res 2000, 60:6266-6271.

25. Jackson TA, Richer JK, Bain DL, Takimoto GS, Tung L, Horwitz KB: The partial agonist activity of antagonist-occupied steroid receptors is controlled by a novel hinge domain-binding coactivator L7/SPA and the corepressors N-CoR or SMRT. Mol Endocrinol 1997, 11:693-705.

26. Rowan BG, O'Malley BW: Progesterone receptor coactivators. Steroids 2000, 65:545-549.

27. Smith CL, Onate SA, Tsai MJ, O'Malley BW: CREB binding protein acts synergistically with steroid receptor coactivator-1 to enhance steroid receptor-dependent transcription. Proc Natl Acad Sci USA 1996, 93:8884-8888.

28. Verrijdt G, Haelens A, Schoenmakers E, Rombauts W, Claessens $F$ : Comparative analysis of the influence of the high-mobility group box 1 protein on DNA binding and transcriptional activation by the androgen, glucocorticoid, progesterone and mineralocorticoid receptors. Biochem J 2002, 361:97-103.

29. Brady ME, Ozanne DM, Gaughan L, Waite I, Cook S, Neal DE Robson CN: Tip60 is a nuclear hormone receptor coactivator. J Biol Chem 1999, 274:17599-17604.

30. Zhou D, Quach KM, Yang C, Lee SY, Pohajdak B, Chen S: PNRC: a proline-rich nuclear receptor coregulatory protein that modulates transcriptional activation of multiple nuclear receptors including orphan receptors SF1 (steroidogenic factor 1) and ERRalpha1 (estrogen related receptor alpha-1). Mol Endocrinol 2000, 14:986-998.

31. Ma ZQ, Liu Z, Ngan ES, Tsai SY: Cdc25B functions as a novel coactivator for the steroid receptors. Mol Cell Biol 2001, 21: 8056-8067.

32. Ko L, Cardona GR, Henrion-Caude A, Chin WW: Identification and characterization of a tissue-specific coactivator, GT198, that interacts with the DNA-binding domains of nuclear receptors. Mol Cell Biol 2002, 22:357-369.

33. Li H, Leo C, Schroen DJ, Chen JD: Characterization of receptor interaction and transcriptional repression by the corepressor SMRT. Mol Endocrinol 1997, 11:2025-2037.

34. Martin AM, Weber BL: Genetic and hormonal risk factors in breast cancer. J Natl Cancer Inst 2000, 92:1126-1135

35. Zheng L, Annab LA, Afshari CA, Lee WH, Boyer TG: BRCA1 mediates ligand-independent transcriptional repression of the estrogen receptor. Proc Natl Acad Sci USA 2001, 98:95879592.

36. Fan M, Long X, Bailey JA, Reed CA, Osborne E, Gize EA, Kirk EA, Bigsby RM, Nephew KP: The activating enzyme of NEDD8 inhibits steroid receptor function. Mol Endocrinol 2002, 16: 315-330.

37. Rubinchik S, Wang D, Yu H, Fan F, Luo M, Norris JS, Dong JY: A negative regulator for the human ER. Mol Endocrinol 2002, 16: 459-468. 